genotype and BMI, FEV1 was inversely associated with $\mathrm{HbA1c}$, $\mathrm{B}=-5.0$ (95\% CI $-6.0-3.0, \mathrm{p}<0.0001$ ).

Conclusion In this large UK data set, an additional $6.6 \%$ of CF patients aged 16-23 years would be diagnosed with diabetes based on HbA1c values. Furthermore, the prevalence of undiagnosed pre-diabetes was high across all age groups and associated with lower \%FEV1.

\section{P195 PROSPECTIVE EXAMINATION OF THE EFFECTS OF IVACAFTOR ON GLYCAEMIC HEALTH}

A Banerjee, AL Brennan, AR Horsley, PJ Barry. Manchester Adult Cystic Fibrosis Centre, Manchester, UK

\subsection{6/thoraxjnl-2014-206260.324}

Background The clinical benefits of the novel cystic fibrosis transmembrane conductance regulator (CFTR) have now been well established for patients carrying the G551D mutation through both phase 3 and real world clinical studies. Modulation of CFTR alters intestinal $\mathrm{pH}$, which may assist in the function of pancreatic enzymes and which theoretically might have an impact on the absorption of nutrients in cystic fibrosis (CF). This may have significant impact on the glycaemic health of patients and early reports from a phase 2 study suggested a significant risk of hyperglycaemia in a patient with pre-existing diabetes.

Aim We aimed to prospectively assess the impact of ivacaftor on glycaemic health

Methods We conducted a prospective observational cohort study of subjects who commenced ivacaftor following NHS approval. Baseline measures were recorded including spirometric measures, weight and sweat chloride. Glycaemic control was assessed using $\mathrm{HbA} 1 \mathrm{c}$ and repeated measures were recorded at 1, 3 and 6 months. Results 24 subjects were included in the study. 17 subjects had normal glucose handling as defined by oral glucose tolerance test, 4 subjects had a pre-existing diagnosis of CF-related diabetes and 3 subjects had impaired glucose tolerance prior to ivacaftor commencement. Ivacaftor significantly increased $\mathrm{FEV}_{1}$ and BMI at 1,3 and 6 months compared to baseline, and decreased sweat chloride at 2 months, all indicating effective CFTR modulation.

There was a significant reduction in $\mathrm{HbA} 1 \mathrm{c}$ from baseline to 6 months in the total cohort, (median $42.5 \mathrm{mmol} / \mathrm{L}$ versus 39.5 $\mathrm{mmol} / \mathrm{L}, \mathrm{p}=0.004)$, but not at other time points. In the diabetic or IGT subgroups, there were no clinically significant changes in HbA1c.

Conclusion Ivacaftor is an effective treatment for CF patients carrying the G551D mutation. In normoglycaemic patients, Ivacaftor significantly reduces HbA1c at 6 months. There was no adverse effect on glucose control noted in diabetic or impaired glucose tolerance subgroups. This may be attributable to improved insulin secretion by CFTR related mechanisms or improved insulin sensitivity. These results are important and reassuring when commencing patients with diabetes on CFTR modulators.

\section{P196 THE EFFECT OF IVACAFTOR THERAPY ON THE MICROBIAL DIVERSITY OF CYSTIC FIBROSIS LUNG INFECTION}

${ }^{1} \mathrm{HD}$ Green, ${ }^{1} \mathrm{PJ}$ Barry, ${ }^{2} \mathrm{C}$ Paisey, ${ }^{2} \mathrm{~A}$ Smith, ${ }^{1}$ WG Flight, ${ }^{2} \mathrm{~J}$ Marchesi, ${ }^{1} \mathrm{AM}$ Jones, ${ }^{1} \mathrm{~A}$ Horsley, ${ }^{2} E$ Mahenthiralingam. 'Manchester Adult Cystic Fibrosis Centre, University Hospital of South Manchester, Manchester, UK; ${ }^{2}$ Cardiff School of Biosciences, Cardiff University, Cardiff, UK

10.1136/thoraxjnl-2014-206260.325
Introduction and objectives Ivacaftor is a CFTR potentiator which is licensed for cystic fibrosis (CF) patients with the G551D mutation. Ivacaftor has led to significant benefits in lung function and weight, a reduction in pulmonary exacerbations and a decrease in time spent on intravenous antibiotics. This impact on exacerbations may be secondary to qualitative or quantitative change in the airway microbiome. The aim of this study was to investigate whether partially restoring CFTR function using Ivacaftor is associated with early changes in airway microbiology.

Methods Paired sputum samples were obtained from 13 adult CF patients immediately prior to Ivacaftor therapy, and after 1 and/or 3 months of treatment. $\mathrm{FEV}_{1}$ was measured at each visit, and sweat chloride was assessed pre-treatment and at 2 months. Samples underwent routine microbiology and extraction of total nucleic acids using a standardised automated method. Ribosomal Intergenic Spacer Analysis (RISA) qualitatively investigated sputum bacterial diversity and 16s rRNA gene pyrosequencing was used to investigate bacterial diversity semi-quantitatively.

Results All subjects had samples at baseline and at either 1 or 3 months post Ivacaftor therapy. 4 subjects had samples at all three time points. Mean FEV1 percent predicted improved from 56 to $63 \%$ at 1 month $(\mathrm{p}<0.01)$. Mean sweat chloride improved from 115 to $54 \mathrm{mmol} / \mathrm{L}(\mathrm{p}<0.01)$. Culture and pyrosequencing analysis showed 11 out of 13 patients had a single dominant infecting pathogen.

These techniques demonstrated no major changes in microbial diversity, especially with regards to the dominant pathogen, preand post-treatment (see Figure 1). 10 patients had a reduction in the number of pyrosequencing reads attributable to Streptococcus on follow-up samples $(\mathrm{p}<0.05)$.

Conclusions Ivacaftor resulted in significant clinical improvements in this group of adult patients within the first 3 months of therapy. Airway microbiology in these patients was largely unaltered in the 3 months after starting Ivacaftor. The preliminary finding of a reduction in Streptococcus reads requires quantitative follow up to evaluate its significance. These findings suggest that potentiation of CFTR function using Ivacaftor does not significantly alter the lung microbiome and clinical improvements witnessed are likely secondary to a different mechanism.

\section{P197 THE INCIDENCE OF NEW PSEUDOMONAS AERUGINOSA INFECTION IN CHILDREN WITH CYSTIC FIBROSIS}

${ }^{1} \mathrm{FJ}$ Gilchrist, ${ }^{2} \mathrm{~J}$ Belcher, ${ }^{3} \mathrm{AM}$ Jones, ${ }^{2} \mathrm{D}$ Smith, ${ }^{4}$ Ar Smyth, ${ }^{5} \mathrm{KW}$ Southern, ${ }^{2} \mathrm{P}$ Spanel, ${ }^{3} \mathrm{AK}$ Webb, ${ }^{1}$ W Lenney. ${ }^{1}$ University Hospital of North Staffordshire, Stoke on Trent, UK; ${ }^{2}$ Keele University, Keele, UK; ${ }^{3}$ Manchester Adult Cystic Fibrosis Centre, Manchester, UK; ${ }^{4}$ Queen's Medical Centre, Nottingham, UK; ${ }^{5}$ Alder Hey Children's Hospital, Liverpool, UK

\subsection{6/thoraxjnl-2014-206260.326}

Introduction Pseudomonas aeruginosa $(\mathrm{PA})$ is one of the most important pathogens in cystic fibrosis (CF). Although there is a wealth of data about the prevalence of chronic PA infection, there is a paucity of evidence about the incidence of new PA infection.

Methods The SPACE (Sensitivity and specificity of PA detection using the hydrogen Cyanide concentration of Exhaled breath) study investigated if exhaled breath hydrogen cyanide is an early marker of PA infection in children with CF. Breath samples, clinical data and microbiology samples were collected at each outpatient appointment from a large cohort of children with CF who had not isolated PA for $>12$ months. This abstract reports the PA acquisition data. 